PlastOx 2007 (2009) 117-129

(C) EDP Sciences, 2009

DOI: $10.1051 /$ ptox/2009009

\title{
Formulation éléments finis des modèles de champ de phases basée sur la théorie de l'équilibre des microforces
}

\author{
K. Ammar ${ }^{1}$, B. Appolaire ${ }^{2}$, G. Cailletaud ${ }^{1}$, F. Feyel ${ }^{1,3}$ et S. Forest ${ }^{1}$ \\ ${ }^{1}$ Mines Paris, ParisTech, Centre des Matériaux / CNRS UMR 7633, BP. 87, \\ 91003 Evry Cedex, France \\ ${ }^{2}$ LSG2M, École des Mines de Nancy, Parc de Saurupt, 54042 Nancy Cedex, France \\ ${ }^{3}$ ONERA, DMSE LCME, 29 Ave. Div. Leclerc, BP. 72, 92322 Châtillon, France
}

\begin{abstract}
Résumé. Nous présentons une formulation éléments finie des modèles de champ de phases pour les alliages binaires, développée dans le cadre général de la thermodynamique. Cette formulation est basée sur la théorie de l'équilibre des microforces, proposée par Gurtin [1]. En utilisant les principes de la thermodynamique des processus irréversibles, les lois de comportement et les équations d'évolution sont clairement exposées et séparées dans la formulation. En outre, des conditions initiales et des conditions aux limites en paramètre d'ordre et en concentration ainsi que leurs quantités duales sont énoncées. L'ensemble des équations d'évolution couplées, à savoir l'équation de champ de phases et l'équation de conservation de la masse, est résolu en utilisant la méthode des éléments finis pour la discrétisation spatiale et un schéma implicite des différences finies pour la discrétisation temporelle.

Le modèle est appliqué à l'étude de la croissance des couches d'oxyde dans un système binaire $\mathrm{Zr}-\mathrm{O}$ à 350C. Des calculs 1D montrent une bonne concordance de la cinétique d'oxydation avec des solutions analytiques. De plus, des calculs bidimensionnels, du même processus, ont été faits pour l'étude de la stabilité morphologique d'une couche d'oxyde ondulée afin d'illustrer les avantages de la méthode des éléments finis avec des frontières complexes et des conditions aux limites variées.
\end{abstract}

\section{INTRODUCTION}

Dans le cadre général de la thermodynamique des milieux continus, nous proposons une formulation éléments finis des modèles de champ de phases pour les alliages binaires. Cette formulation est basée sur la théorie de l'équilibre des microforces, proposée par Gurtin [1], où une équation supplémentaire d'équilibre, fonction du paramètre d'ordre et de son gradient, est introduite.

La formulation est employée pour simuler les évolutions morphologiques complexes des microstructures hétérogènes (formes, répartition des précipités et croissance de grains...) et décrire l'interface diffuse d'épaiseur non nulle entre deux phases. En utilisant les différents principes de la thermodynamique, nous déterminons les lois d'état et les équations constitutives, qui sont clairement séparées dans la formulation, de sorte que des modèles non-linéaires et fortement couplés puissent être implantés plus facilement dans un code des éléments finis. En conséquence, la dérivation de ces relations d'état peut être généralisée pour une large classe de comportements des matériaux en présence des processus dissipatifs tels que le transfert thermique et la mécanique, et plus particulièrement la plasticité, afin de prévoir les contraintes induites par les transformations de phase. Cette formulation peut être appliquée aux corps finis périodiques et non périodiques, aux microstructures hétérogènes, dans laquelle des conditions initiales et des conditions aux limites en paramètre d'ordre et en concentration ainsi que leurs quantités duales sont clairement énoncées.

Dans la littérature, diverses méthodes numériques ont été utilisées pour résoudre les équations de champ de phases, telles que les volumes finis, la méthode de Monte-Carlo et la transformée de Fourier, classiquement employée pour des microstructures hétérogènes, principalement avec des conditions périodiques $[2,3]$. Cependant, la méthode des éléments finis représente généralement une manière robuste, bien adaptée au traitement du problème aux limites. D'ailleurs, elle a l'avantage de pouvoir 
s'appliquer à des géométries quelconques, à des conditions aux limites variées, et offre la possibilité d'étudier des nombreuses lois de comportement couplées.

Des travaux précédents ont appliqué la méthode des éléments finis pour l'étude de transformations de phases telles que la résolution de l'équation de Cahn-Hilliard [4], la simulation de la solidification [5] et l'étude des matériaux ferroélectriques [6].

L'objectif de ce travail est de fournir, dans un premier temps, une base nécessaire introductive à la simulation numérique en exhibant une formulation éléments finis des équations de champ de phases; à savoir l'équation de Landau-Ginzburg et l'équation de la diffusion en formulant les conditions aux limites. Dans un second temps, nous présentons l'implémentation numérique du problème aux limites, en appliquant une approximation spatiale et temporelle, comme a été présentée dans [7].

Ce présent modèle est bien adapté à l'étude des modèles à interface diffuse à épaisseur non nulle, dans lequel l'état local d'une microstructure hétérogène est décrit par des champs conservatifs de concentration $c$ et des champs non-conservatifs de paramètre d'ordre $\phi$, associé à la nature des phases. L'évolution temporelle et spatiale de ce champ est régie par l'équation de type Ginzburg-Landau :

$$
\beta \dot{\phi}=\alpha \Delta \phi-\frac{\partial f}{\partial \phi}
$$

Dans un système hétérogène, l'approche de Ginzburg-Landau est basée sur une fonctionnelle d'énergie libre totale, qui s'exprime comme la somme d'une contribution d'une densité d'énergie libre homogène $f_{0}(c, \phi)$ et un terme quadratique en gradient, relié à l'énergie de l'interface :

$$
f(c, \phi, \nabla \phi)=f_{0}(c, \phi)+\frac{\alpha}{2} \nabla \phi \cdot \nabla \phi
$$

Finalement, le modèle est appliqué à l'étude de la cinétique de croissance des couches d'oxyde dans un système $\mathrm{Zr} / \mathrm{ZrO}_{2}$. Le comportement du système métal-oxyde est abordé à travers des modélisations par éléments finis.

\section{2. ÉQUILIBRE DES MICROFORCES ET ÉNONCÉS FONDAMENTAUX DE LA THERMODYNAMIQUE}

\subsection{Principe des puissances virtuelles}

Le principe des puissances virtuelles fournit une méthode fondamentale, fondée sur une approche énergétique, pour dériver les équations d'équilibre et les conditions aux limites dans diverses situations physiques [8,9]. L'application de ce principe, à un domaine isolé $\mathcal{V}$, de surface extérieure $\partial \mathcal{V}$, nécessite la détermination des puissances des efforts appliqués sur le système pour lequel il est commode de poser un champ du paramètre d'ordre virtuel $\phi^{\star}(\underline{\boldsymbol{x}}, t)$.

En se basant sur la théorie de Gurtin [1], nous supposons l'existence d'un système des microforces, défini par le vecteur microcontrainte $\boldsymbol{\xi}$, le scalaire microforce interne $\pi$, le scalaire microforce externe $\gamma$ et le vecteur microforce externe $\gamma$.

La puissance virtuelle des efforts intérieurs se présente comme une intégrale sur le volume $V$, supposée définie par une forme linéaire des densités volumiques des microforces $\pi$ et $\underline{\xi}$ :

$$
\begin{aligned}
\mathcal{P}^{(i)}\left(\phi^{\star}, V\right) & =\int_{V}\left(\pi \phi^{\star}-\underline{\boldsymbol{\xi}} \cdot \nabla \phi^{\star}\right) d v \\
& =\int_{V}(\pi+\nabla \cdot \underline{\boldsymbol{\xi}}) \phi^{\star} d v-\int_{\partial V}(\underline{\boldsymbol{\xi}} \cdot \underline{\boldsymbol{n}}) \phi^{\star} d s
\end{aligned}
$$

La puissance des efforts extérieurs est composée, d'une part, d'une puissance des efforts exercés à distance, supposée définie par une densité volumique de microforces vectorielles $\underline{\gamma}$ ainsi que de 
microforces scalaires $\gamma$,

$$
\begin{aligned}
\mathcal{P}^{(e)}\left(\phi^{\star}, V\right) & =\int_{V}\left(\gamma \phi^{\star}+\underline{\boldsymbol{\gamma}} \cdot \nabla \phi^{\star}\right) d v \\
& =\int_{V}(\gamma-\nabla \cdot \underline{\boldsymbol{\gamma}}) \phi^{\star} d v+\int_{\partial V}(\underline{\boldsymbol{\gamma}} \cdot \underline{\boldsymbol{n}}) \phi^{\star} d s
\end{aligned}
$$

et d'autre part, d'une puissance des efforts de contact, shématisée par une densité surfacique $\zeta$ :

$$
\mathcal{P}^{(c)}\left(\phi^{\star}, V\right)=\int_{\partial V} \zeta \phi^{\star} d s
$$

En négligeant la puissance virtuelle des quantités d'accélération $\left(\mathcal{P}^{(a)}\left(\phi^{\star}, V\right)=0\right)$, la définition des différentes puissances permet à présent d'appliquer le P.P.V. et plus précisément l'axiome de l'équilibre statique, qui postule que, pour tout champ du paramètre d'ordre virtuel $\phi^{\star}$, la puissance virtuelle de tous les efforts appliqués au système, tant intérieurs qu'extérieurs, est nulle, quel que soit le domaine $\mathcal{D} \subset V$ :

$\forall \phi^{\star}, \forall \mathcal{D} \subset V$

$$
\begin{gathered}
\mathcal{P}^{(i)}\left(\phi^{\star}, \mathcal{D}\right)+\mathcal{P}^{(c)}\left(\phi^{\star}, \mathcal{D}\right)+\mathcal{P}^{(e)}\left(\phi^{\star}, \mathcal{D}\right)=0 \\
\int_{\mathcal{D}}(\pi+\nabla \cdot \underline{\boldsymbol{\xi}}+\gamma-\nabla \cdot \underline{\boldsymbol{\gamma}}) \phi^{\star} d v+\int_{\partial \mathcal{D}}(\zeta-\underline{\boldsymbol{\xi}} \cdot \underline{\boldsymbol{n}}+\underline{\boldsymbol{\gamma}} \cdot \underline{\boldsymbol{n}}) \phi^{\star} d s=0
\end{gathered}
$$

Cette identité ne peut être vérifiée, quel que soit $\mathcal{D}$ et pour tout champ $\phi^{\star}$, que si :

$$
\begin{gathered}
\nabla \cdot(\underline{\boldsymbol{\xi}}-\underline{\boldsymbol{\gamma}})+\pi+\gamma=0 \quad \text { in } \quad V \\
\zeta=(\underline{\boldsymbol{\xi}}-\underline{\boldsymbol{\gamma}}) \cdot \underline{\boldsymbol{n}} \quad \text { on } \quad \partial V
\end{gathered}
$$

La première équation (8) traduit la forme générale de l'équation d'équilibre des microforces. Elle correspond à la théorie de Gurtin, excepté le vecteur microforce externe $\gamma$, qui peut exister en général. Tandis que l'équation (9) représente la condition aux limites en paramètre d'ordre. Dans ce travail, nous supposons que les microforces externes sont nulles $(\gamma=0$ et $\underline{\gamma}=0)$.

\subsection{Lois d'état, potentiel de dissipation}

Le premier principe de la thermodynamique, appelé encore principe de conservation d'énergie, stipule que, lors d'une transformation et à chaque instant, la dérivée particulaire de l'énergie totale d'un système est la somme de la puissance des efforts extérieurs exercés sur le système $\mathcal{P}^{(e)}(\underline{\boldsymbol{v}})$ et du taux de chaleur $\mathcal{Q}$. L'énergie totale est repartie en énergie interne et en énergie cinétique $\mathcal{K}$ :

$$
\dot{\mathcal{K}}+\dot{E}=\mathcal{P}^{(e x t)}+\mathcal{Q}
$$

où $\mathcal{Q}$ est l'apport de chaleur au système, $\mathcal{K}$ est l'énergie cinétique et $E$ est l'énergie interne.

En absence des forces d'inertie et pour une transformation isotherme $(\mathcal{Q}=0)$, le principe de conservation d'énergie se réduit à l'expression suivante :

$$
\begin{aligned}
\dot{E} & =\int_{\mathcal{V}} \dot{e} d v=\mathcal{P}^{e x t}=-\mathcal{P}^{i n t} \\
& =\int_{\partial V}(\underline{\boldsymbol{\xi}} \cdot \underline{\boldsymbol{n}}) \dot{\phi} d s=\int_{V} \nabla \cdot(\underline{\boldsymbol{\xi}} \dot{\phi}) d v
\end{aligned}
$$

avec $e$ est la densité d'énergie interne.

Cette identité est vérifiée quel que soit le domaine $\mathcal{D} \subset V$. Ainsi la forme locale du bilan d'énergie s'écrit :

$$
\dot{e}=\nabla \cdot(\dot{\phi} \underline{\xi})
$$


Le deuxième principe de la thermodynamique traduit la non-conservation de l'entropie lors d'une transformation. Il stipule que, pour une transformation isotherme, le taux de production d'entropie est toujours supérieur ou égale au flux d'entropie :

$$
\int_{V} \dot{s} d v \geq-\int_{\partial V} \underline{\boldsymbol{\Phi}} \cdot \underline{\boldsymbol{n}} d s \quad \text { et } \quad \underline{\boldsymbol{\Phi}}=-\mu \frac{\boldsymbol{J}}{T}
$$

avec $s$ est la densité d'entropie, $\underline{\Phi}$ le flux d'entropie, $\underline{\boldsymbol{J}}$ le flux de matière donné par la loi de diffusion et $\mu$ le potentiel chimique.

En utilisant la relation de proportionnalité entre la variation de la quantité d'espèces chimiques dans un volume et le flux de matière, donnée par l'équation de la conservation de masse,

$$
\dot{c}=-\nabla \cdot \underline{J}
$$

nous obtenons la forme locale du deuxième principe de la thermodynamique.

$$
T \dot{s}-\nabla \cdot(\mu \underline{\boldsymbol{J}}) \geq 0
$$

La méthode de l'état local postule que l'état thermodynamique du milieu en un point et à un instant donné est complètement défini par la définition de variables d'état, ne dépendant que du point considéré. L'énergie libre totale du système est un potentiel qui dépend de la concentration $c$, du paramètre d'ordre $\phi$ ainsi que de son gradient $\nabla \phi$. La dérivation de la densité d'énergie libre s'exprime sous la forme :

$$
\begin{aligned}
\dot{f}(c, \phi, \nabla \phi) & =\dot{e}-T \dot{s} \\
& =\frac{\partial f}{\partial c} \dot{c}+\frac{\partial f}{\partial \phi} \dot{\phi}+\frac{\partial f}{\partial \nabla \phi} \cdot \nabla \dot{\phi}
\end{aligned}
$$

La combinaison de l'équation ci-dessus, dans le cas isotherme, avec Eqs. (12)-(15), conduit à l'inégalité de Clausius-Duhem :

$$
\left(\mu-\frac{\partial f}{\partial c}\right) \dot{c}-\left(\pi+\frac{\partial f}{\partial \phi}\right) \dot{\phi}+\left(\underline{\xi}-\frac{\partial f}{\partial \nabla \phi}\right) \cdot \nabla \dot{\phi}-\underline{J} \cdot \nabla \mu \geq 0
$$

Pour chaque processus admissible et pour tous les champs des variables $(c, \phi, \nabla \phi)$, l'inégalité (17) doit être vérifiée quelles que soient les valeurs de $\dot{c}, \dot{\phi}$ et $\nabla \dot{\phi}$. Sachant que la densité d'énergie libre $f$, la microcontrainte vectorielle $\underline{\xi}$, les microforces scalaires $\pi$ et le potentiel chimique $\mu$ dépendent des variables d'état :

$$
(f, \underline{\xi}, \mu)=\text { fonction de }(c, \phi, \nabla \phi)
$$

On admet toutefois une dépendance $\pi(c, \phi, \dot{\phi})$ afin de rendre compte de processus dissipatifs. Ainsi, $\dot{c}$ et $\nabla \dot{\phi}$ apparaissent linéairement dans l'inégalité de Clausius-Duhem, cela implique [10,11] :

$$
\begin{gathered}
\mu=\frac{\partial f}{\partial c}=\frac{\partial f_{0}}{\partial c} \\
\underline{\boldsymbol{\xi}}=\frac{\partial f}{\partial \boldsymbol{\nabla} \phi}=\alpha \boldsymbol{\nabla} \phi
\end{gathered}
$$

Ces relations définissent les lois d'état de modèle.

En adoptant la forme (2) pour la densité d'énergie libre, l'inégalité de Clausius-Duhem se réduit à l'expression de la dissipation suivante, qui doit être positive :

$$
D=-\underline{J} \cdot \nabla \mu-\pi_{d i s} \dot{\phi} \geq 0 \quad \text { et } \quad \pi_{d i s}=\pi+\frac{\partial f}{\partial \phi}
$$

avec $\pi_{d i s}$ est une microforce chimique, associée aux processus dissipatifs, introduite dans [1]. 
Pour définir les lois complémentaires relatives aux processus dissipatifs, nous postulons l'existence d'un potentiel de dissipation, se présentant comme une fonction de $\left(\nabla \mu\right.$ et $\left.\pi_{d i s}\right)$ et qui s'exprime comme suit :

$$
\Omega\left(\nabla \mu, \pi_{d i s}\right)=\frac{1}{2} L(\phi) \nabla \mu \cdot \nabla \mu+\frac{1}{2}(1 / \beta) \pi_{d i s}^{2}
$$

avec $L(\phi)$ et $\beta$ sont des paramètres matériaux. La convexité du potentiel de dissipation assure la positivité de la dissipation.

Les lois complémentaires d'évolution sont définies alors, à partir de ce potentiel de dissipation, par les relations suivantes :

$$
\begin{gathered}
\dot{\phi}=-\frac{\partial \Omega}{\partial \pi_{d i s}}=-(1 / \beta) \pi_{d i s} \\
\underline{\boldsymbol{J}}=-\frac{\partial \Omega}{\partial \nabla \mu}=-L(\phi) \nabla \mu
\end{gathered}
$$

L'utilisation combinée des (21) et (23) permet de réécrire la première loi complémentaire d'évolution comme suit:

$$
\pi=-\beta \dot{\phi}-\frac{\partial f}{\partial \phi}
$$

La substitution des deux lois d'état et des lois complémentaires d'évolution dans l'équation de conservation de masse et l'équation de l'équilibre des microforces permet de déduire respectivement l'équation d'évolution de la concentration et du paramètre d'ordre :

$$
\begin{gathered}
\dot{c}=-\nabla \cdot(-L(\phi) \nabla \mu)=-\nabla \cdot\left(-L(\phi) \nabla \frac{\partial f}{\partial c}\right) \\
\nabla \cdot \underline{\xi}+\pi=-\beta \dot{\phi}+\alpha \Delta \phi-\frac{\partial f}{\partial \phi}=0
\end{gathered}
$$

L'équation classique de la diffusion et l'équation de Ginzburg-Landau sont ainsi obtenues.

\section{RÉSOLUTION NUMÉRIQUE}

Dans cette partie, nous présentons la méthode des éléments finis appliquée pour résoudre numériquement le problème. Dans un premier temps, nous construirons, à partir des équations issues de ce modèle, un problème faible associé. En particulier, nous exhiberons les formulations variationnelles de l'équation de la diffusion (26) et de l'équation d'équilibre des microforces (27), en utilisant les conditions aux limites. Dans un second temps, nous décrirons l'implémentation numérique en appliquant une approximation spatiale par éléments finis et une approximation temporelle par différences finies.

\subsection{Formulation variationnelle}

La formulation variationnelle de l'equation différentielle de champ de phases peut être découlée directement du principe des puissances virtuelles (6) :

$$
\Im\left(\phi^{\star}\right)=\int_{V}\left(\pi \phi^{\star}-\underline{\xi} \cdot \nabla \phi^{\star}\right) d v+\int_{\partial V} \zeta \phi^{\star} d s=0
$$

Pour déduire une formulation faible de l'équation (26), il reste à préciser les conditions appliquées sur la frontière en concentration. Par conséquent, nous choisissons une condition aux limites en flux de matière $\mathrm{j}$, définie par :

$$
j=\underline{\boldsymbol{J}} \cdot \underline{\boldsymbol{n}} \quad \text { sur } \quad \partial \mathcal{V}
$$


Compte tenu de cette condition, la formulation variationnelle de l'équation de la diffusion s'écrit sous la forme suivante :

$$
\Im\left(c^{\star}\right)=\int_{V}\left(\dot{c} c^{\star}-\underline{\boldsymbol{J}} \cdot \nabla c^{\star}\right) d v+\int_{\partial V} j c^{\star} d s=0
$$

avec $c^{\star}$ est un champ arbitraire de concentration virtuelle.

Il s'ensuit que le modèle discret couplé champ de phases/diffusion, en régime transitoire, peut être formulé comme suit :

Touver $\{c(\underline{\boldsymbol{x}}, t), \phi(\underline{\boldsymbol{x}}, t)\}$ vérifiant :

$$
\mid \begin{aligned}
& \text { à } \quad t=0 \\
& c(\underline{\boldsymbol{x}}, 0)=c_{0}(\underline{\boldsymbol{x}}) \\
& \phi(\underline{\boldsymbol{x}}, 0)=\phi_{0}(\underline{\boldsymbol{x}}) \\
& \text { à tout instant } t>0 \\
& \Im\left(c^{\star}\right)=\int_{V}\left(\dot{c} c^{\star}-\underline{\boldsymbol{J}} \cdot \nabla c^{\star}\right) d v+\int_{\partial V} j c^{\star} d s=0 \\
& \Im\left(\phi^{\star}\right)=\int_{V}\left(\pi \phi^{\star}-\underline{\boldsymbol{\xi}} \cdot \nabla \phi^{\star}\right) d v+\int_{\partial V} \zeta \phi^{\star} d s=0
\end{aligned}
$$

\subsection{Discrétisation}

La discrétisation en espace utilisée pour notre problème est de type éléments finis. Le domaine spatial $\mathcal{V}$ est subdivisé en $N$ éléments et la solution continue du problème sera approchée dans l'espace par un nombre fini de valeurs aux nœuds. Les champs de concentration $c(\underline{\boldsymbol{x}}, t)$ et de paramètre d'ordre $\phi(\underline{\boldsymbol{x}}, t)$, qui représentent les deux degrés de liberté, sont estimés à l'intérieur de chaque élément du maillage et à chaque instant $t$, à partir des valeurs qu'ils prennent aux nœuds associés à cet élément en faisant intervenir des fonctions de forme de l'élément $N^{e}(\underline{\boldsymbol{x}})$ :

$$
\begin{array}{ll}
c(\underline{\boldsymbol{x}}, t)=\sum_{i=1}^{n} N_{i}^{e}(\underline{\boldsymbol{x}}) c_{i}(t), \quad \phi(\underline{\boldsymbol{x}}, t)=\sum_{i=1}^{n} N_{i}^{e}(\underline{\boldsymbol{x}}) \phi_{i}(t) \\
c^{\star}(\underline{\boldsymbol{x}}, t)=\sum_{i=1}^{n} N_{i}^{e}(\underline{\boldsymbol{x}}) c_{i}^{\star}(t), \quad \phi^{\star}(\underline{\boldsymbol{x}}, t)=\sum_{i=1}^{n} N_{i}^{e}(\underline{\boldsymbol{x}}) \phi_{i}^{\star}(t) \\
\nabla c(\underline{\boldsymbol{x}}, t)=\sum_{i=1}^{n} B_{i}^{e}(\underline{\boldsymbol{x}}) c_{i}(t), \quad \nabla \phi(\underline{\boldsymbol{x}}, t)=\sum_{i=1}^{n} B_{i}^{e}(\underline{\boldsymbol{x}}) \phi_{i}(t)
\end{array}
$$

où $n$ est le nombre des nœuds de l'élément $e$ et $\left[B^{e}(\underline{\boldsymbol{x}})\right]$ est la matrice faisant intervenir les dérivées des fonctions de forme. Elle s'écrit, dans le cas à deux dimensions, sous la forme suivante :

$$
\left[B^{e}(\underline{\boldsymbol{x}})\right]=\left[\begin{array}{cccc}
\frac{\partial N_{1}^{e}}{\partial x} & \frac{\partial N_{2}^{e}}{\partial x} & \cdots & \frac{\partial N_{n}^{e}}{\partial x} \\
\frac{\partial N_{1}^{e}}{\partial y} & \frac{\partial N_{2}^{e}}{\partial y} & \cdots & \frac{\partial N_{n}^{e}}{\partial y}
\end{array}\right]
$$

La résolution du système des équations continues nécessite, outre l'approximation spatiale, une approximation temporelle puisque les deux degrés de liberté $c$ et $\phi$ dépendent du temps $t$. La discrétisation temporelle est obtenue en utilisant un schéma d'Euler implicite par la méthode des différence finies. Par conséquent, nous choisissons deux conditions initiales en concentration $c_{0}$ et en paramètre d'ordre $\phi_{0}$ et nous estimons les solutions approchées à chaque instant par des pas de temps successifs $\Delta t$. Les discrétisations temporelles $\dot{c}(t+\Delta t)$ et $\dot{\phi}(t+\Delta t)$ peuvent ainsi être estimées en résolvant les équations suivantes :

$$
\begin{gathered}
\dot{c}(t+\Delta t)=\frac{c(t+\Delta t)-c(t)}{\Delta t} \\
\dot{\phi}(t+\Delta t)=\frac{\phi(t+\Delta t)-\phi(t)}{\Delta t} \\
c(0)=c_{0}, \quad \phi(0)=\phi_{0}
\end{gathered}
$$


La substitution de la discrétisation spatiale et temporelle dans le système (31) permet de déduire le vecteur résidu élémentaire total $\left\{R^{e}(c, \phi)\right\}$. C'est la quantité que nous cherchons à annuler en tout point du volume et à tout instant, tout en respectant les conditions aux limites choisies.

$$
\left\{R^{e}(c)\right\}=\left\{\begin{array}{l}
R_{c}^{e}(c) \\
R_{\phi}^{e}(\phi)
\end{array}\right\}
$$

où $R_{c}^{e}(c)$ et $R_{\phi}^{e}(\phi)$ sont les résidus élémentaires, spécifiques à chaque élément, et qui s'expriment respectivement en fonction de la concentration $c$ et du paramètre d'ordre $\phi$.

$$
\begin{aligned}
& \left(R_{c}^{e}\right)_{i}=\int_{V^{e}} N_{i}^{e} N_{j}^{e} \dot{c}_{j}^{e}-\left[B^{e}\right]_{i j} J_{j} d v+\int_{\partial V^{e}} N_{i}^{e} j d s \\
& \left(R_{\phi}^{e}\right)_{i}=\int_{V^{e}} N_{i}^{e} \pi(\phi)-\left[B^{e}\right]_{i j} \xi_{j} d v+\int_{\partial V^{e}} N_{i}^{e} \zeta d s
\end{aligned}
$$

Le vecteur résidu global est obtenu par assemblage des quantités élémentaires en utilisant la matrice d'assemblage $\left[A^{e}\right]$ :

$$
\{R(\phi)\}=\sum_{e=1}^{N}\left[A^{e}\right] \cdot\left\{R^{e}(\phi)\right\}=\{0\}
$$

Pour résoudre ce système non-linéaire, nous avons recours à la méthode de Newthon-Raphson, dont nous avons besoin de définir la matrice tangente. Cette matrice élémentaire $\left[K_{t}^{e}\right]$, spécifique à chaque élément e, est obtenue par dérivation du vecteur résidu élémentaire (36) par rapport aux champs des variables :

$$
\begin{gathered}
{\left[K_{t}^{e}\right]=\left[\frac{\partial R^{e}}{\partial \delta^{e}}\right]=\left[\begin{array}{c}
{\left[K_{c c}^{e}\right]\left[K_{c \phi}^{e}\right]} \\
{\left[K_{\phi c}^{e}\right]\left[K_{\phi \phi}^{e}\right]}
\end{array}\right]} \\
\text { with }\left\{\delta^{e}\right\}=\left\{\begin{array}{l}
\left\{c^{e}\right\} \\
\left\{\phi^{e}\right\}
\end{array}\right\}
\end{gathered}
$$

où $\left(K_{c c}^{e}\right)_{i j},\left(K_{c \phi}^{e}\right)_{i j},\left(K_{\phi c}^{e}\right)_{i j}$ et $\left(K_{\phi \phi}^{e}\right)_{i j}$ sont les composantes des matrices tangentes, qui s'écrivent de la façon suivante :

$$
\begin{gathered}
\left(K_{c c}^{e}\right)_{i j}=\frac{\partial\left(R_{c}^{e}\right)_{i}}{\partial c_{j}^{e}}=\int_{V^{e}} \frac{1}{\Delta t} N_{i}^{e} N_{j}^{e}-\left[B^{e}\right]_{i k}\left[\frac{\partial \underline{\boldsymbol{J}}}{\partial c^{e}}\right]_{k j} d v \\
\left(K_{c \phi}^{e}\right)_{i j}=\frac{\partial\left(R_{c}^{e}\right)_{i}}{\partial \phi_{j}^{e}}=\int_{V^{e}}-\left[B^{e}\right]_{i k}\left[\frac{\partial \underline{\boldsymbol{J}}}{\partial \phi^{e}}\right]_{k j} d v \\
\left(K_{\phi c}^{e}\right)_{i j}=\frac{\partial\left(R_{\phi}^{e}\right)_{i}}{\partial c_{j}^{e}}=\int_{V^{e}} N_{i}^{e}\left(\frac{\partial \pi}{\partial c^{e}}\right)_{j} d v \\
\left(K_{\phi \phi}^{e}\right)_{i j}=\frac{\partial\left(R_{\phi}^{e}\right)_{i}}{\partial \phi_{j}^{e}}=\int_{V^{e}} N_{i}^{e}\left(\frac{\partial \pi}{\partial \phi^{e}}\right)_{j}-\left[B^{e}\right]_{i k}\left[\frac{\partial \underline{\xi}}{\partial \phi^{e}}\right]_{k j} d v
\end{gathered}
$$

La matrice tangente globale $\left[K_{t}(\phi)\right]$ est obtenue par assemblage des matrices élémentaires tangentes $\left[K_{t}^{e}(\phi)\right]$ de tous les éléments par l'intermédiaire de la matrice d'assemblage.

Les éléments utilisés, dans ce travail, sont des éléments linéaires ou quadratiques avec intégration réduite (des triangles à 3 ou 6 points de Gauss et des quadrangles à 4 ou 8 points de Gauss ...) [7]. 


\section{APPLICATION À L'OXYDATION DU ZIRCONIUM}

\subsection{Paramètres}

Le choix d'une fonctionnelle d'énergie libre est un point capital dans les modèles de champ de phases puisqu'il détermine non seulement le type du système que nous cherchons à étudier mais également l'efficacité des calculs et la convergence de la méthode numérique employée pour résoudre les équations aux dérivées partielles.

Dans la littérature, nous trouvons plusieurs fonctionnelles d'énergie libre telles que la fonctionnelle de Khachaturyan pour les alliages, le modèle de Cahn pour l'étude de la décomposition spinoïdale et l'énergie libre à potentiel polynomial pour l'étude des alliages binaires, qui a été proposée par Kim et al. [12] et que nous adoptons dans ce travail. L'avantage essentiel de ce modèle est qu'il est thermodynamiquement admissible, c'est-à-dire que les équations d'évolutions s'expriment en fonction des dérivées variationnelles de l'énergie libre du système. Ainsi, nous obtenons des équations de champ de phases plus simples, caractérisées par une relation linéaire du potentiel chimique en fonction de la concentration et du paramètre d'ordre.

En effet, la densité d'énergie libre est exprimée comme la somme d'une contribution d'une fonction $g(\phi)$ de type "double puits" pénalisant énergétiquement l'interface et d'une énergie homogène $f_{0}(c, \phi)$. Cette dernière se présente comme l'interpolation des énergies libres des deux phases $\left(f_{1}, f_{2}\right)$, par l'intermédiaire d'une fonction polynomiale $h(\phi)$.

$$
f_{0}(c, \phi)=\psi(c, \phi)+W g(\phi) \quad \text { avec } \quad \psi(c, \phi)=h(\phi) f_{1}(c)+[1-h(\phi)] f_{2}(c)
$$

avec $W$ est la hauteur de la barrière d'énergie entre les deux phases.

Les polynômes spécifiques $g(\phi)=\phi^{2}(1-\phi)^{2}$ et $h(\phi)=\phi^{2}(3-2 \phi)$ ont été choisis comme dans [13].

Pour simplifier, les densités d'énergie libre des deux phases ont été décrites par une forme quadratique, fonction seulement de la concentration [14] :

$$
f_{i}(c)=\frac{1}{2} k_{i}\left(c-A_{i}\right)^{2} \quad \text { avec } \quad i=\{1,2\}
$$

Les constantes $k_{1}, k_{2}$ représentent les courbures des énergies libres de chacune des phases, décrites par des paraboles. Elles sont positives afin d'éviter le cas de la décomposition spiroïdale. $A_{1}, A_{2}$ sont les concentrations d'équilibre des deux phases $\alpha$ et $\beta$, données par la courbe de coexistence du diagramme de phase et correspondentes aux minima des $f_{1}$ et $f_{2}$.

Les paramètres de champ de phases $\alpha$ et $W$ peuvent être reliés à l'énergie et l'épaisseur de l'interface, respectivement $\sigma$ et $\delta$. S'intéresser au profil d'équilibre d'une interface plane, nous permet d'expliciter comment ces paramètres apparaissent dans la méthode de champ de phases.

En effet, à l'équilibre thermodynamique entre deux phases, $\dot{\phi}=0$, l'équation (1), le long de l'interface plane, devient :

$$
\alpha \frac{d^{2} \phi_{\mathrm{eq}}}{d x^{2}}=\frac{\partial f_{0}}{\partial \phi_{\mathrm{eq}}}
$$

En intégrant analytiquement cette équation différentielle, nous obtenons le profil d'équilibre d'une interface plane $\phi_{\mathrm{eq}}(x)$ :

$$
\phi_{\text {eq }}=1-\tanh \left(\frac{x}{\delta}\right) \quad \text { avec } \delta=2.94 \sqrt{2 \alpha / W}
$$

où le facteur 2.94 vient de la manière dont l'épaisseur d'interface $\delta$ a été définie, c.-à-d. pour des valeurs de $\phi$ dans la gamme [0.05; 0.95], représentant la longueur sur laquelle $\delta$ varie de manière significative. 
Tableau 1. Paramètres de calcul pour le système zirconium/oxygène à $350^{\circ} \mathrm{C}$.

\begin{tabular}{lcc}
\hline \hline$\beta\left(\mathrm{J} \mathrm{s} / \mathrm{m}^{3}\right)$ & $1.78 \times 10^{5}$ \\
$\sigma\left(\mathrm{J} / \mathrm{m}^{2}\right)$ & 0.1 \\
$\delta(\mathrm{m})$ & \multicolumn{2}{c}{$7 \times 10^{-8}$} \\
$\alpha(\mathrm{J} / \mathrm{m})$ & \multicolumn{2}{c}{$7.14 \times 10^{-9}$} \\
$W\left(\mathrm{~J} / \mathrm{m}^{3}\right)$ & \multicolumn{2}{c}{$2.5 \times 10^{7}$} \\
$V_{m}\left(\mathrm{~m}^{3} /\right.$ mole $)$ & $\mathrm{Zr}(i=1)$ & \multicolumn{2}{c}{$10^{-5}$} & $\mathrm{ZrO}_{2}(i=2)$ \\
\hline phase & 0.24 & 0.66 \\
$\mathrm{~A}_{i}($ fraction molaire $)$ & 1 & 1 \\
$\mathrm{k}_{i} V_{m}(\mathrm{~J} /$ mole $)$ & $1.722 \times 10^{-20}$ & $6.368 \times 10^{-18}$ \\
$\mathrm{D}_{i}\left(\mathrm{~m}^{2} / \mathrm{s}\right)$ de $[17]$ & & \\
\hline \hline
\end{tabular}

Généralement, il y a deux contributions dans l'énergie d'interface : la première découle de la fonction double puits $g(\phi)$, et la seconde vient de la variation de la concentration dans l'interface [15].

$$
\sigma=\sqrt{\alpha W} /(3 \sqrt{2})
$$

Le coefficient d'Onsager $L(\phi)$ est défini comme une interpolation des coefficients de diffusion de chaque phase $D_{1}$ et $D_{2}$ par l'intermédiaire de la fonction $h(\phi)$ :

$$
L(\phi)=h(\phi) D_{1} / k_{1}+(1-h(\phi)) D_{2} / k_{2} .
$$

\subsection{Oxydation du zirconium}

Le modèle de champ de phases est implémenté dans le code de calcul par éléments finis ZeBuLon et employé pour l'étude de la croissance des couches d'oxyde à $350^{\circ} \mathrm{C}$ dans le système binaire $\mathrm{Zr}-\mathrm{O}$.

Les paramètres utilisés sont donnés dans le tableau (1). Une énergie d'interface de $100 \mathrm{~mJ} / \mathrm{m}^{2}$, a été choisie, elle correspond à une interface cohérente/semi-cohérente entre l'oxyde et le zirconium [16].

Nous utilisons un maillage uniforme unidimensionnel d'une longueur de $1 \mu \mathrm{m}$, avec 1000 éléments quadrangles linéaires (4 points de Gauss). Les conditions aux limites en paramètre d'ordre et en concentration, imposées sur les deux facettes du système, sont :

$$
\begin{array}{llll}
\underline{\boldsymbol{\xi}} \cdot \underline{\boldsymbol{n}}=0 & \text { et } & \boldsymbol{J} \cdot \underline{\boldsymbol{n}}=0 & \text { sur la surface droite } \\
\underline{\boldsymbol{\xi}} \cdot \underline{\boldsymbol{n}}=0 & \text { et } & \text { sur la surface gauche }
\end{array}
$$

La condition de Dirichlet en concentration $(c=0.68)$, imposée sur la surface gauche, a été choisie légèrement supérieure à la concentration stœchiométrique de l'oxygène afin d'accélérer la réaction de l'oxyde sur le métal.

Un profil initial en tangente hyperbolique de $\phi$ et $c$, correspondant à une couche initiale d'oxyde $\left(\mathrm{ZrO}_{2}\right)$ d'une épaisseur de $84 \mathrm{~nm}$, a été placé à la surface externe d'une plaque de métal (zirconium). Nous choisissons une concentration initiale de zirconium de 0.22 , proche de la concentration d'équilibre, correspondante à une zone de sous-saturation en oxygène dans la phase $\alpha$ (Fig. 1).

Les profils de concentration sont présentés à différentes heures dans la (figure 1). Au début du processus (après $2 \mathrm{~h}$ ), les concentrations à l'interface sont légèrement plus hautes que celle à l'équilibre $(c=0.66)$. Ainsi, un gradient d'oxygène se développe dans la phase $\alpha$, provoqué par la croissance de l'oxyde. Le processus de la croissance est ainsi gouverné par le phénomène de diffusion de l'oxygène, à travers la couche d'oxyde, dans les deux phases. Une partie de l'oxygène diffuse dans le métal et une autre sert à la croissance de la couche. Très rapidement, la saturation est définitivement atteinte en tout point de l'interface en équilibre local, mais l'oxygène n'ayant pas le temps de diffuser dans le métal vu que la formation de l'oxyde à l'interface métal-oxyde est rapide devant le phénomène de diffusion.

La croissance de la couche d'oxyde, présentée sur la (figure 2), la suit une cinétique parabolique c-à-d. $\Delta e=K \sqrt{t}$. La constante de croissance $K=7.5 \cdot 10^{-10} \mathrm{~m} / \mathrm{s}$ a été déterminée par une régression 


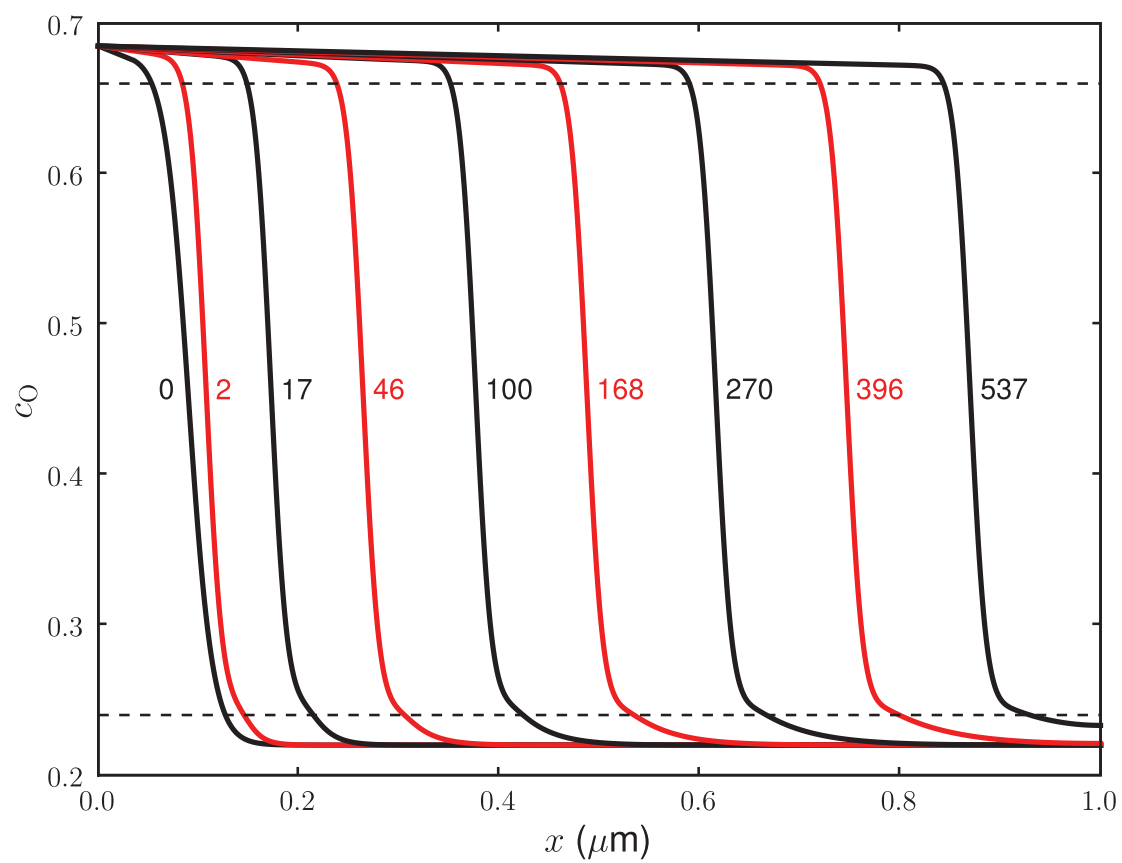

Figure 1. Évolution du profil de concentration en oxygène; les nombres sur les courbes correspondent au temps de calcul en heures et les deux lignes discontinues horizontales sont les concentrations d'équilibre.

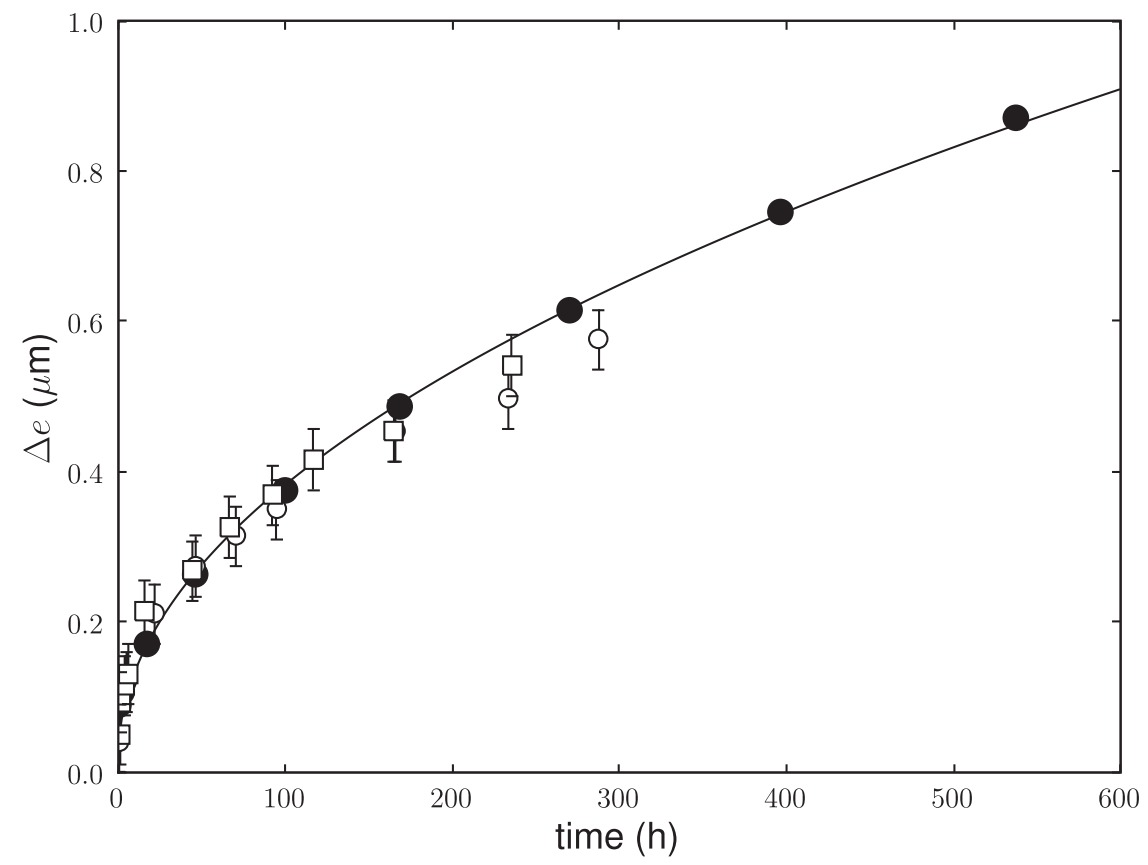

Figure 2. La cinétique de croissance de la couche d'oxyde : les points sont liés aux profils de la Fig. 1 et la ligne continue correspond au meilleur ajustement d'une loi parabolique. Des mesures expérimentales réalisées dans [17] pour le Zircaloy-4 ont été superposées avec des symboles blancs. 

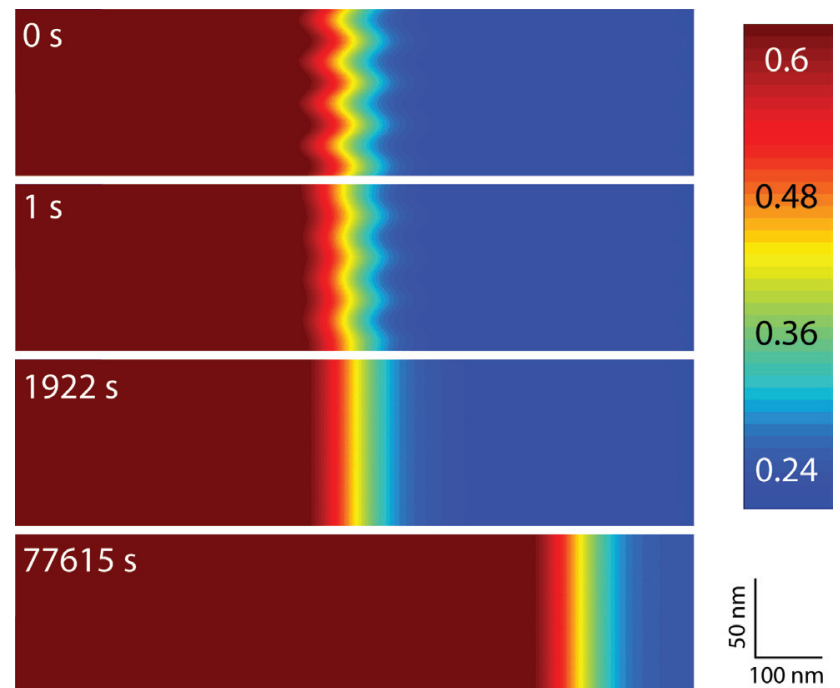

Figure 3. Évolution temporelle du champ de concentration en oxygène durant la croissance d'une couche d'oxyde avec une interface ondulée.
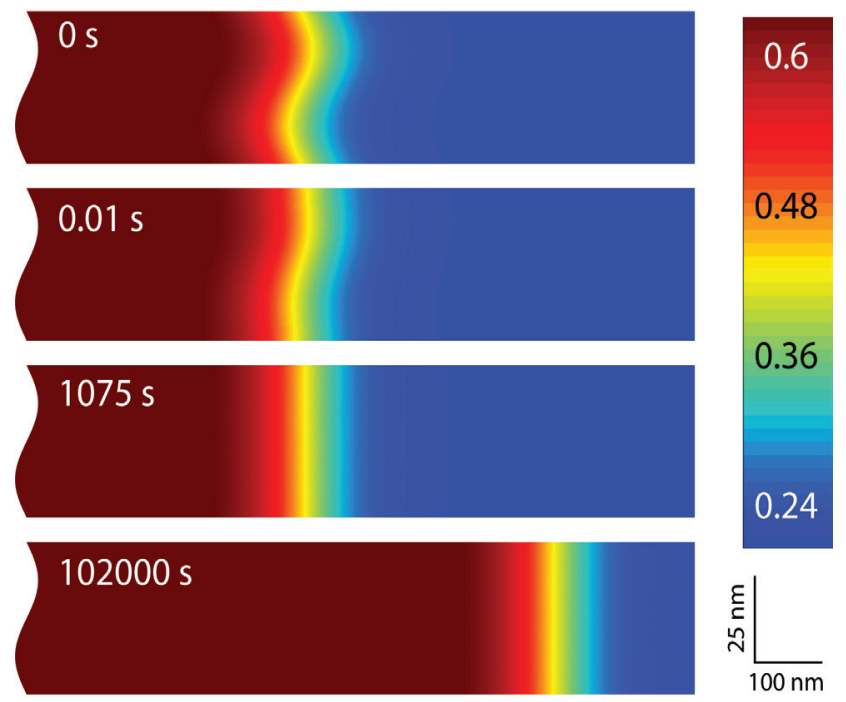

Figure 4. Évolution temporelle du champ de concentration en oxygène durant la croissance d'une couche sinusoïdale d'oxyde.

linéaire des couples des données $(\ln (\Delta e), \ln (t))$, excluant les premiers points qui sont fortement influencés par les conditions initiales. Cette constante est en bon accord avec la valeur $7.75 \cdot 10^{-10} \mathrm{~m} / \mathrm{s}$, déterminée analytiquement [18]. En outre, la figure montre une bonne concordance entre les résultats numériques obtenues et les mesures expérimentales réalisées dans [17].

Afin d'illustrer l'avantage de la méthode des éléments finis pour la résolution du modèle de champ de phases sur des géométries quelconques, des calculs bidimensionnels ont été faits pour étudier la cinétique d'oxydation afin d'illustrer l'influence de la géométrie de l'interface et de la surface externe sur la stabilité morphologique de la couche d'oxyde. Par conséquent, des petites ondulations (en sinus) 
ont été imposées initialement soit sur l'interface métal/oxyde (fig. 3), ou sur la couche d'oxyde (fig. 4), avec différentes longueurs d'onde. Dans les deux cas, nous observons que l'ondulation de l'interface ont complètement disparu après mille secondes, c.-à-d. sur une courte échelle de temps par rapport au processus de la croissance. Ces résultats sont aussi conformes à l'analyse analytique d'un problème semblable traité dans [18].

\section{CONCLUSION}

Une formulation par éléments finis des modèles de champ de phases pour les alliages binaires a été présentée, basée sur la théorie de l'équilibre des microforces et le cadre général de la thermodynamique des processus irréversibles. Utilisant la méthode des éléments finis pour la discrétisation spatiale et la méthode des différences finies pour la discrétisation temporelle, des simulations numériques ont été faites pour étudier la cinétique d'oxydation du zirconium pur. Des autres validations ont été effectuées pour différents nombres de nœuds et plusieurs types de maillage (libre et régulier...) avec des éléments linéaires et quadratiques. En outre, divers cas ont été étudiés en modifiant, par exemple, les courbures et les hauteurs des énergies libres des deux phases, qui n'ont pas été rapportés ici.

La formulation, présentée ici, permet, d'une part, l'application de toutes les formes générales des énergies libres, telle que l'énergie de Folch-Plapp [19] et le modèle de Khachaturyan [20], et d'autre part, l'utilisation des corps finis, des géométries quelconques avec des conditions aux limites variées périodiques ou non-périodiques. En plus, une extension de ce modèle sera obtenue en présence d'autres processus dissipatifs, tel que le couplage avec la mécanique, et plus particulièrement la plasticité.

\section{Références}

[1] Gurtin M.E. Physica D, 92 (1996) 178.

[2] Bronchart Q. Développement de méthodes de champs de phase quantitatives et applications à la précipitions homogène dans les alliages binaires. Thèse de doctorat, Université de CergyPontoise, 2006.

[3] Chen L.Q. and Hu S.Y. Phase-field method applied to strain-dominated microstructure evolution during solid-state phase transformations. In D. Raabe, F. Roters, F. Barlat, and L.Q. Chen, editors, Continuum Scale Simulation of Engineering Materials, pages 271-296. Wiley-VCH, 2004.

[4] Chan P.K. and Rey A.D. Computational Materials Sciences, 3 (1995) 377.

[5] Danilov D. and Nestler B. J. Crystal Growth, 275 (2005) 177.

[6] Schrade D., Mueller R., Xu B.X., and Gross D. Computer Methods in Applied Mechanics and Engineering, 196 (2007) 4365.

[7] Besson J., Cailletaud G., Chaboche J.-L. and Forest S.. Mécanique non linéaire des matériaux. Hermès Sciences, France, 2001.

[8] Germain. P. J. de Mécanique, 12 (1973) 235.

[9] Germain P. SIAM J. Appl. Math., 25 (1973) 556.

[10] Coleman B.D. and Noll W. Arch. Rational Mech. and Anal., 13 (1963) 167.

[11] Coleman B.D. and Gurtin M.E. J. Chemical Physics, 47 (1967) 597.

[12] Kim S.G., Kim W.T. and Suzuki T. Phys. Rev. E, 60 (1999) 7186.

[13] Wang S.-L., Sekerka R.F., Wheeler A.A., Murray B.T., Coriell S.R., Braun R.J. and McFadden G.B. Physica D, 69 (1993) 189.

[14] Echebarria B., Folch R., Karma A. and Plapp M. Phys. Rev. E, 70 (2004) 061604.

[15] Kim S.G., Kim W.T. and Suzuki T. Phys. Rev. E, 58 (1998) 3316.

[16] Penelle R., Boisot P., Béranger G. and Lacombe P. J. Nucl. Mater., 30 (1971) 340. 
[17] Parise M. Mécanismes de corrosion des alliages de zirconium : Etude des cinétiques initiales d'oxydation et du comportement mécanique du système métal-oxyde. Thèse de doctorat, Ecole des Mines de Paris, 1996.

[18] Appolaire B. and Gouné M. Computational Materials Science, 38 (2006) 126.

[19] Folch R. and Plapp M. Phys. Rev. E, 72 (2005) 011602.

[20] Wang Y. and Khachaturyan A.G. Acta Metall. Mater., 43 (1995) 1837. 
